\title{
Parity reversion in real interest rate in the Asian countries: further evidence based on local-persistent model
}

\begin{abstract}
This paper examines the validity of real interest parity (RIP) for 10 Asian economies over the period 1977-2012 (quarterly frequency). The evidence based on two-break unit root tests reveals that majority of the real interest rate differentials (RIDs) with respect to Germany and the US are stationary, but this appears not to be the case for the Japan-based RIDs. Contrary to these results, the point estimates and the confidence intervals (CIs) of half-lives based on the Phillips et al.'s (2001) local-persistent model provide a clear-cut conclusion on RIP: Most of the RIDs take less than a year to adjust back to their respective equilibrium values, with notably tighter CIs than what has been suggested by earlier literature.
\end{abstract}

Keyword: Real interest rate parity; Structural breaks; Half-lives; Local-persistent model 